\title{
Signal Propagation in Transmission Lines with Losses Using Fibonacci Wave Functions
}

\author{
Simon Hissem and Lamine Mamadou Doumbia
}

\begin{abstract}
In this paper, the general model for an infinite LC ladder network using Fibonacci wave functions that were applied to lossless transmission lines will be extended to transmission lines including losses. The general model that was derived from a first order system transfer function representing a simple RC or RL circuit will be used to model and analyze transmission lines presenting losses. The LC ladder network model can be applied to any order for each inductor current with its parasitic $r_{L}$ resistor and for each capacitor voltage with its parasitic $r_{C}$ resistor. The extension of the proposed general model to transmission lines with losses is subject to Heaviside condition for both resistors $r_{C}$ and $r_{L}$.
\end{abstract}

Keywords - Fibonacci wave functions, Heaviside, LC ladder, losses, Pascal's triangle, Transmissions lines.

\section{INTRODUCTION}

In the previous papers [1] and [2], Fibonacci wave functions (FWFs) were introduced to model an infinite LC ladder network without losses. A general model for any LC ladder network order was also introduced.

In the literature, very few papers analyzed either LC ladder or transmission cable including losses with exhaustive mathematical calculations [4]. Most other papers used only lossless modeling and analysis. Introducing losses in such systems leads to another level of difficulties in modelling and analyzing these systems [3], [5] and [6]. Fibonacci wave functions may help simplify and extend the analysis to another horizon.

In this paper, the extension of FWFs with its general model to LC ladder network with losses to model a transmission lines with resistive losses will be modeled and analyzed for different orders and will be compared to MATLAB-Simulink LC model presenting the same losses. The paper is organized as follow. Sections II and III are dedicated to the general model extension to resistive-capacitive including losses Fibonacci electrical circuit (RC-FEC-Losses) and resistive inductive including losses Fibonacci electrical circuit (RLFEC-Losses. Section IV describes a comparative study of FWFs and RC-FEC-Losses using MATLAB-Simulink model. Section V shows a comparative study of FWFs and RC-FEC-Losses of transmission line with short and open terminations.

\section{RC-FEC-LOSSES FIBONACCI ELECTRICAL CIRCUIT}

The original Fibonacci transfer function has the following form [1].

$$
g_{1}^{\left(k, x_{c}\right)}(s)=\frac{K}{s+x_{c}}
$$

with

$$
K=\omega_{f}^{2} \text { and } x_{c}=\frac{1}{R C}
$$

The first order electrical circuit RC-FEC-Losses is presented in Fig. 1.

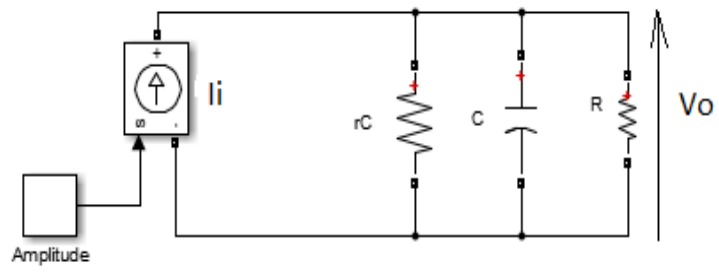

Fig. 1. First order RC-FEC-Losses

The equivalent impedance between the capacitor $\mathrm{C}$ and its parasitic resistor $r_{c}$ can be easily calculated as follows.

$$
Z_{c}=\frac{\frac{1}{C}}{s+\frac{1}{r_{C} C}}
$$

with $a=\frac{1}{r_{C} C}$ and changing Laplace variable $\mathrm{s}$ to the new reference $\delta=s+a$. The impedance $Z_{c}$ becomes.

$$
Z_{c}=\frac{1}{S C}
$$

Furthermore, the circuit in figure 1 can easily be written in the new Laplace reference defined by Laplace variable $\mathcal{S}$

$$
\begin{gathered}
\frac{V_{o}}{I_{i}}=\frac{\frac{1}{C}}{\mathcal{S}+\frac{1}{R C}}=L \frac{\frac{1}{L C}}{\mathcal{S}+\frac{1}{R C}}=L \frac{K}{\mathcal{S}+x_{c}}=L G_{1}^{\left(K, x_{c}, a\right)}(\mathcal{S}) \\
\left(\frac{V_{o}}{L I_{i}}\right)_{1}^{\left(K, x_{c}, a\right)}=\frac{K}{\mathcal{S}+x_{c}}=G_{1}^{\left(K, x_{c}, a\right)}(\mathcal{S}) \\
K=\frac{1}{L C} \text { and } x_{c}=\frac{1}{R C}
\end{gathered}
$$

The second order RC-FEC-Losses circuit diagram shown in Fig. 2 and its transfer function in (3).

Mamadou Lamine Doumbia, Université du Québec à Trois-Rivières, Trois-Rivières, Québec, Canada.

(e-mail :mamadou.doumbia@uqtr.ca).
Submitted on September 14, 2021

Published on October 06, 2021.

Simon Hissem, Higher College of Technology, Abu-Dhabi, UAE.

(e-mail: shissem@hct.ac.ae) 


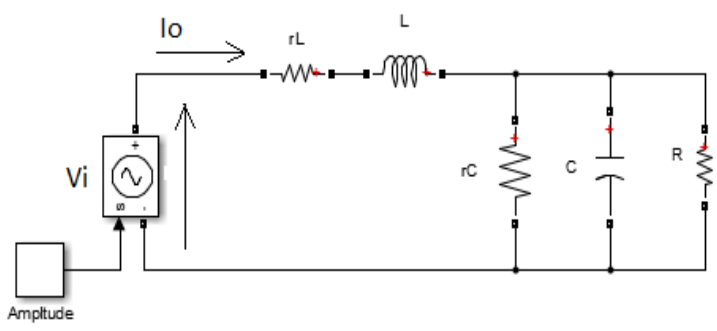

Fig. 2. Second order RC-FEC-Losses.

The equivalent impedance between the inductor $\mathrm{L}$ and its parasitic resistor $r_{L}$ can be easily calculated as follows:

$$
Z_{L}=r_{L}+s L=\left(s+\frac{r_{L}}{L}\right) L
$$

with $a_{L}=\frac{r_{L}}{L}$ and changing Laplace variable to the new reference $\delta=s+a_{L}$ with the condition $a_{L}=a$ which is exactly the known Heaviside condition for no signal distortion through the transmission lines. This condition does not eliminate losses through the lines. This condition is necessary to extend the Fibonacci wave functions to transmission lines that present losses.

$$
a=\frac{r_{L}}{L}=\frac{1}{r_{C} C} \text { with } \mathcal{S}=s+a
$$

The impedance $Z_{L}$ becomes $Z_{L}=\delta L$.

Furthermore, the circuit in figure 2 can easily be written in the new Laplace reference defined by Laplace variable $S_{a}$.

$$
\begin{gathered}
V_{i}=\left(L G_{1}^{\left(K, x_{c}, a\right)}(\mathcal{S})+\mathcal{S} L\right) I_{o}=\frac{L C\left(\mathcal{S}+G_{1}^{\left(K, x_{c}, a\right)}(\mathcal{S})\right) I_{o}}{C} \\
\left(\frac{I_{o}}{C V_{i}}\right)_{2}^{\left(K, x_{c}, a\right)}=\frac{K}{\mathcal{S}+\frac{K}{\mathcal{S}+x_{c}}}=G_{2}^{\left(K, x_{c}, a\right)}(\mathcal{S})
\end{gathered}
$$

The transfer function of the third order RC-FEC-Losses (5) is derived from circuit diagram presented in Fig. 3.

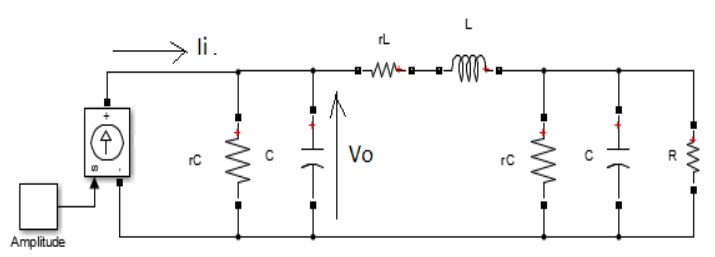

Fig. 3. Third order RC-FEC-losses

$$
\begin{gathered}
I_{i}=\left(C G_{2}^{\left(K, x_{c}, a\right)}(\mathcal{S})+\mathcal{S} C\right) V_{o}=\frac{L C\left(\mathcal{S}+G_{2}^{\left(K, x_{c}, a\right)}(\mathcal{S})\right) V_{o}}{L} \\
\left(\frac{V_{o}}{L I_{i}}\right)_{3}^{\left(K, x_{c}, a\right)}=\frac{K}{\mathcal{S}+\frac{K}{\mathcal{S}+\frac{K}{\mathcal{S}+x_{c}}}}=G_{3}^{\left(K, x_{c}, a\right)(s)}
\end{gathered}
$$

One can see that an even $n^{\text {th }}$ order RC-FEC-Losses (Fig. 4) will have voltage as input and current as output.

$$
n=n_{c}+n_{L}
$$

$\mathrm{n}_{\mathrm{c}}$ total number of capacitors in the circuit.
$\mathrm{n}_{\mathrm{L}}$ total number of inductances in the circuit.

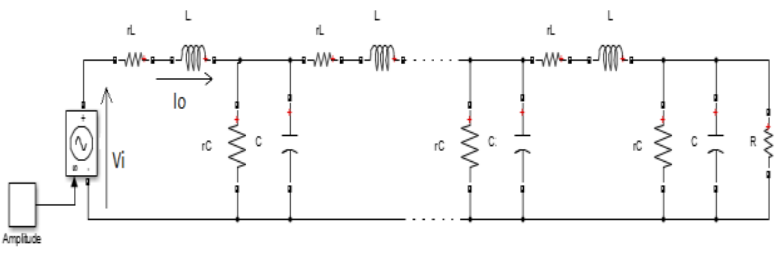

Fig. 4. $n^{\text {th }}$ even order RC-FEC-Losses.

The FWF of the $n^{\text {th }}$ even order of the circuit in Fig. 4 is:

$$
\left(\frac{I_{o}}{C V_{i}}\right)_{n}^{\left(K, x_{c}, a\right)}=\frac{K}{\mathcal{S}+G_{n-1}^{\left(K, x_{c}, a\right)}(\mathcal{S})}=G_{n}^{\left(K, x_{c}, a\right)}(\mathcal{S})
$$

For the $n^{\text {th }}$ odd order in the circuit in Fig. 5, the FWF is:

\begin{tabular}{|c|c|}
\hline \multicolumn{2}{|c|}{$\mathcal{L}(\mathcal{S}, a)$} \\
\hline$\left(\frac{V_{o}}{L I_{i}}\right)_{1}^{\left(K, x_{c}, a\right)}=G_{1}^{\left(K, x_{c}, a\right)}=\frac{K}{\mathcal{S}+\boldsymbol{x}_{\boldsymbol{c}}}$ & $\left(\frac{V_{o}}{L I_{i}}\right)_{1}^{\left(K, x_{c}\right)}=G_{1}^{\left(K, x_{c}, a\right)}=\frac{K}{1 \mathcal{S}+1 \boldsymbol{x}_{\boldsymbol{c}}}$ \\
\hline $\begin{array}{l}\left(\frac{I_{o}}{C V_{i}}\right)_{2}^{\left(K, x_{c}, a\right)}=G_{2}^{\left(K, x_{c}, a\right)} \\
=\frac{K}{\mathcal{S}+\frac{K}{\mathcal{S}+\boldsymbol{x}_{\boldsymbol{c}}}}\end{array}$ & $\begin{array}{l}\left(\frac{I_{o}}{C V_{i}}\right)_{2}^{\left(K, x_{c}, a\right)}=G_{2}^{\left(K, x_{c}, a\right)} \\
=\frac{K \mathcal{S}+K \boldsymbol{x}_{\boldsymbol{c}}}{1 \mathcal{S}^{2}+1 \boldsymbol{x}_{c} \mathcal{S}+1 \boldsymbol{K}}\end{array}$ \\
\hline $\begin{array}{l}\left(\frac{V_{o}}{L I_{i}}\right)_{3}^{\left(K, x_{c}, a\right)}=G_{3}^{\left(K, x_{c}, a\right)} \\
=\frac{K}{\mathcal{S}+\frac{K}{\mathcal{S}+\frac{K}{\mathcal{S}+\boldsymbol{x}_{\boldsymbol{c}}}}}\end{array}$ & $\begin{array}{l}\left(\frac{V_{o}}{L I_{i}}\right)_{3}^{\left(K, x_{c}, a\right)}=G_{3}^{\left(K, x_{c}, a\right)} \\
=\frac{K \mathcal{S}^{2}+K \mathcal{S} \boldsymbol{x}_{\boldsymbol{c}}+K^{2}}{1 \mathcal{S}^{3}+1 \boldsymbol{x}_{\boldsymbol{c}} \mathcal{S}^{2}+2 \boldsymbol{K} \mathcal{S}+1 \boldsymbol{K} \boldsymbol{x}_{\boldsymbol{c}}}\end{array}$ \\
\hline $\begin{array}{l}\left(\frac{I_{o}}{C V_{i}}\right)_{4}^{\left(K, x_{c}, a\right)}=G_{4}^{\left(K, x_{c}, a\right)} \\
=\frac{K}{\mathcal{S}+\frac{K}{\mathcal{S}+\frac{K}{\mathcal{S}+\frac{K}{\mathcal{S}+\boldsymbol{x}_{\boldsymbol{c}}}}}}\end{array}$ & $\begin{array}{l}\left(\frac{I_{o}}{C V_{i}}\right)_{4}^{\left(K, x_{c}, a\right)}=G_{4}^{\left(K, x_{c}, a\right)} \\
=\frac{K \mathcal{S}^{3}+K \boldsymbol{x}_{\boldsymbol{c}} \mathcal{S}^{2}+2 K^{2} \mathcal{S}+K^{2} \boldsymbol{x}_{\boldsymbol{c}}}{1 \mathcal{S}^{4}+1 \boldsymbol{x}_{\boldsymbol{c}} \mathcal{S}^{3}+3 \boldsymbol{K} \mathcal{S}^{2}+2 \boldsymbol{K} \boldsymbol{x}_{\boldsymbol{c}} \mathcal{S}+1 \boldsymbol{K}^{2}}\end{array}$ \\
\hline 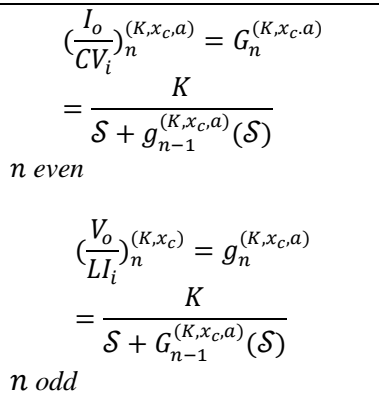 & $\begin{array}{l}g_{n}^{\left(K, x_{c}, a\right)}(\mathcal{S}) \\
=\frac{K \operatorname{den}_{n-1}^{\left(K, x_{c}, a\right)}(\mathcal{S})}{\mathcal{S d e n}_{n-1}^{\left(K, x_{c}, a\right)}(\mathcal{S})+\operatorname{num}_{n-1}^{\left(K, x_{c}, a\right)}(\mathcal{S})} \\
G_{n}^{\left(K, x_{c}, a\right)}(\mathcal{S})=\frac{K \operatorname{den}_{n-1}^{\left(K, x_{c}, a\right)}(\mathcal{S})}{\operatorname{den}_{n}^{\left(K, x_{c}, a\right)}(\mathcal{S})}\end{array}$ \\
\hline
\end{tabular}

$$
\left(\frac{V_{o}}{L I_{i}}\right)_{n}^{\left(K, x_{c}, a\right)}=\frac{K}{s+G_{n-1}^{\left(K, x_{c}, a\right)}(\mathcal{S})}=G_{n}^{\left(K, x_{c}, a\right)}(\mathcal{S})
$$

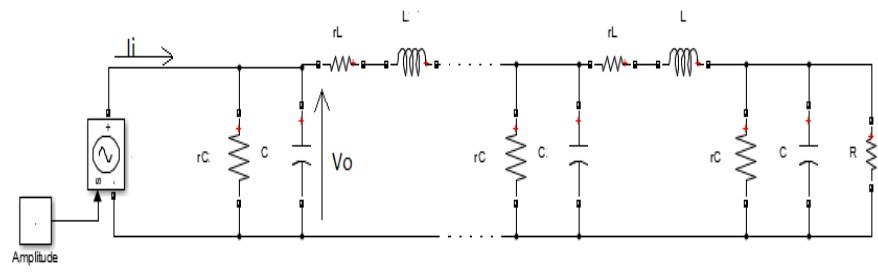

Fig. 5. $n^{\text {th }}$ odd order RC-FEC-Losses.

Table I shows general FWFs in RC-FEC-Losses for all orders.

TABLE I: RC-FEC-Losses FibonAcCi WAVE FunCtIONS IN REFERENCE 
All currents through each inductor and voltages through each capacitor are determined by their corresponding FWFs. For $n^{\text {th }}=40$ of RC-FEC-Losses in Fig. 6, its general model can be easily generated with all FWFs for all currents in each inductor and all voltages in each capacitors in Fig. 7.

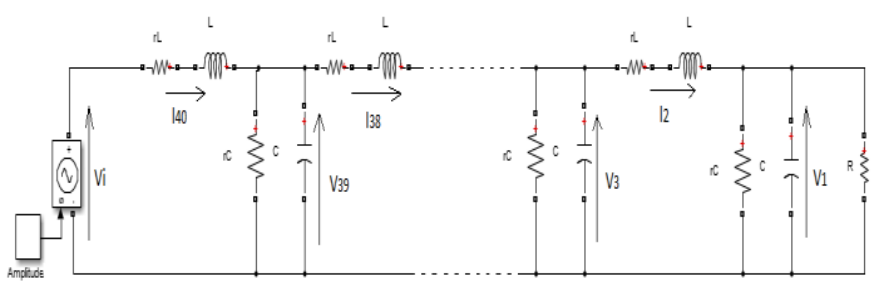

Fig. 6. $n^{\text {th }}$ even order RC-FEC-Losses.
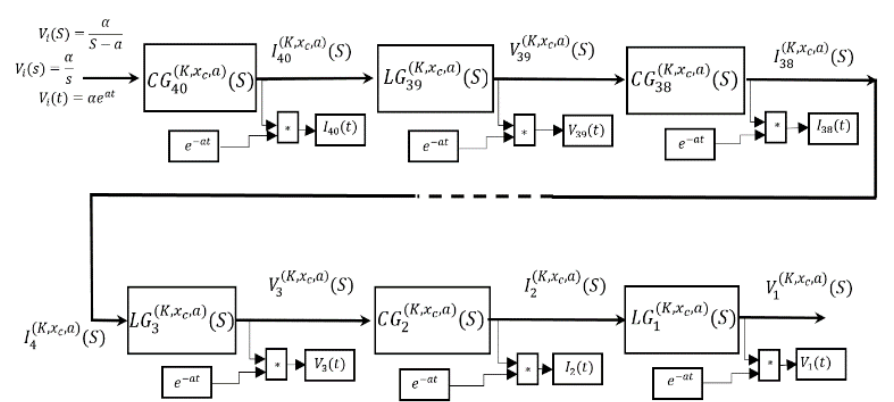

Fig. 7. $n^{\text {th }}=40$ even order RC-FEC-Losses Model using FWFs for each current and voltage branch in reference $\mathcal{L}(\mathcal{S}, a)$.

\section{RL-FEC-LOSSES FIBONACCI ELECTRICAL CIRCUIT}

As RC-FEC-Losses Fibonacci wave functions FWFs, RLFEC-Losses can be calculated easily. The first order circuit in Fig. 8 and its FWF is presented in (9).

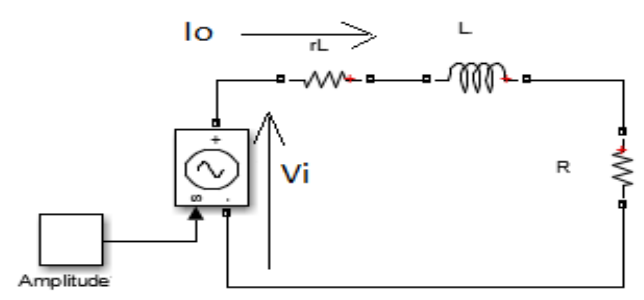

Fig. 8. First order RL-FEC-Losses.

$$
\begin{gathered}
\left(\frac{I_{o}}{C V_{i}}\right)_{1}^{\left(K, x_{L}, a\right)}=\frac{K}{\mathcal{S}+x_{L}}=G_{1}^{\left(K, x_{L}, a\right)}(\mathcal{S}) \\
K=\frac{1}{L C} \text { and } x_{L}=\frac{R}{L} \\
K=\frac{1}{L C}
\end{gathered}
$$
(10).

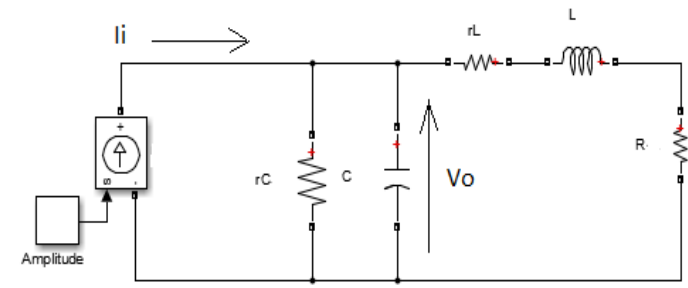

Fig. 9. Second order RL-FEC-Losses

$$
\left(\frac{V_{o}}{L I_{i}}\right)_{2}^{\left(K, x_{L}, a\right)}=\frac{K}{\mathcal{S}+\frac{K}{\mathcal{S}+x_{L}}}=G_{2}^{\left(K, x_{L}, a\right)}(\mathcal{S})
$$

The third order RL-FEC-Losses will be defined with an input voltage and output current (Fig. 10) and its FTF in (11)

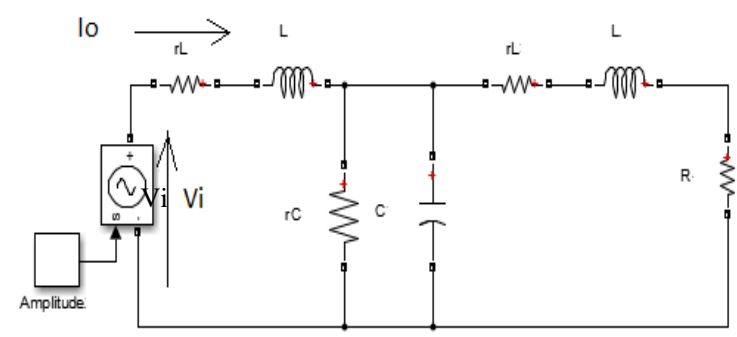

Fig. 10. First order RL-FEC-Losses.

$$
\left(\frac{I_{o}}{C V_{i}}\right)_{3}^{\left(K, x_{L}, a\right)}=\frac{K}{\mathcal{S}+\frac{K}{\mathcal{S}+\frac{K}{\mathcal{S}+x_{L}}}}=G_{3}^{\left(K, x_{L}, a\right)}(\mathcal{S})
$$

an even $n^{\text {th }}$ order RL-FEC-Losses in figure 11 has its FWF expressed in (13).

$$
n=n_{c}+n_{L}
$$

$\mathrm{n}_{\mathrm{c}}$ is the total number of capacitors in the circuit.

$\mathrm{n}_{\mathrm{L}}$ is the total number of inductance in the circuit.

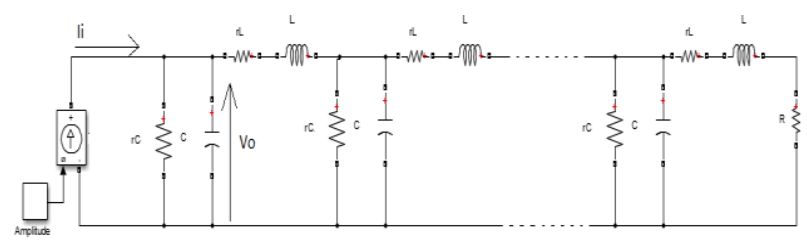

Fig. 11. $n^{\text {th }}$ even order RL-FEC-Losses.

$$
\left(\frac{V_{o}}{L I_{i}}\right)_{n}^{\left(K, x_{L}, a\right)}=\frac{K}{\mathcal{S}+G_{n-1}^{\left(K, x_{L}\right)}(\mathcal{S})}=G_{n}^{\left(K, x_{L}, a\right)}(\mathcal{S})
$$

An odd $n^{\text {th }}$ order RL-FEC-Losses in Fig. 12 has its FWF expressed in (14).

$$
\left(\frac{I_{o}}{C V_{i}}\right)_{n}^{\left(K, x_{L}, a\right)}=\frac{K}{\mathcal{S}+G_{n-1}^{\left(K, x_{L}, a\right)}(\mathcal{S})}=G_{n}^{\left(K, x_{L}, a\right)}(\mathcal{S})
$$

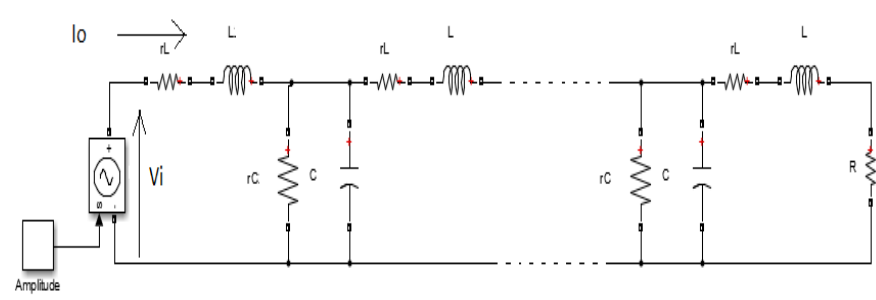

Fig. 12. $n^{\text {th }}$ odd order RL-FEC-Losses.

Table II shows FWFs in the case of RL-FEC-Losses with losses. 
TABLE II: RL-FEC-LOSSES FIBONACCI WAVE FUNCTIONS IN REFERENCE $\mathcal{L}(\mathcal{S}, a)$

\begin{tabular}{|c|c|}
\hline $\begin{array}{l}\left(\frac{I_{o}}{L V_{i}}\right)_{1}^{\left(K, x_{L}, a\right)}=G_{1}^{\left(K, x_{L}, a\right)} \\
=\frac{K}{\mathcal{S}+x_{L}}\end{array}$ & $\left(\frac{I_{o}}{L V_{i}}\right)_{1}^{\left(K, x_{L}, a\right)}=G_{1}^{\left(K, x_{L}, a\right)}=\frac{K}{1 \mathcal{S}+1 x_{\boldsymbol{L}}}$ \\
\hline $\begin{array}{l}\left(\frac{V_{o}}{L I_{i}}\right)_{2}^{\left(K, x_{L}, a\right)}=G_{2}^{\left(K, x_{L}, a\right)} \\
=\frac{K}{\mathcal{S}+\frac{K}{\mathcal{S}+x_{L}}}\end{array}$ & $\begin{aligned}\left(\frac{V_{o}}{L I_{i}}\right)_{2}^{\left(K, x_{L}, a\right)}=G_{2}^{\left(K, x_{L}, a\right)} & \\
& =\frac{K \mathcal{S}+K \boldsymbol{x}_{\boldsymbol{L}}}{1 \mathcal{S}^{2}+1 \boldsymbol{x}_{\boldsymbol{L}} \mathcal{S}+1 \boldsymbol{K}}\end{aligned}$ \\
\hline $\begin{array}{l}\left(\frac{I_{o}}{L V_{i}}\right)_{3}^{\left(K, x_{L}, a\right)}=G_{3}^{\left(K, x_{L}, a\right)} \\
=\frac{K}{\mathcal{S}+\frac{K}{\mathcal{S}+\frac{K}{\mathcal{S}+x_{L}}}}\end{array}$ & $\begin{array}{l}\left(\frac{I_{o}}{L V_{i}}\right)_{3}^{\left(K, x_{L}, a\right)}=G_{3}^{\left(K, x_{L}, a\right)} \\
=\frac{K \mathcal{S}^{2}+K \mathcal{S} \boldsymbol{x}_{\boldsymbol{L}}+K^{2}}{1 \mathcal{S}^{3}+1 \boldsymbol{x}_{\boldsymbol{L}} \mathcal{S}^{2}+2 \boldsymbol{K} \mathcal{S}+1 \boldsymbol{K} \boldsymbol{x}_{\boldsymbol{L}}}\end{array}$ \\
\hline $\begin{array}{l}\left(\frac{V_{o}}{L I_{i}}\right)_{4}^{\left(K, x_{L}, a\right)}=G_{4}^{\left(K, x_{L}, a\right)} \\
=\frac{K}{\mathcal{S}+\frac{K}{\mathcal{S}+\frac{K}{\mathcal{S}+\frac{K}{\mathcal{S}+\boldsymbol{x}_{\boldsymbol{L}}}}}}\end{array}$ & $\begin{array}{l}\left(\frac{V_{o}}{L I_{i}}\right)_{4}^{\left(K, x_{L}, a\right)}=G_{4}^{\left(K, x_{L}, a\right)} \\
=\frac{K \mathcal{S}^{3}+K \boldsymbol{x}_{L} \mathcal{S}^{2}+2 K^{2} \mathcal{S}+K^{2} \boldsymbol{x}_{\boldsymbol{L}}}{1 \mathcal{S}^{4}+1 \boldsymbol{x}_{\boldsymbol{L}} \mathcal{S}+3 \boldsymbol{K} \mathcal{S}^{2}+2 \boldsymbol{K} \boldsymbol{x}_{\boldsymbol{L}} \mathcal{S}+1 \boldsymbol{K}^{2}}\end{array}$ \\
\hline $\begin{array}{l}\text { n even } \\
\left(\frac{V_{o}}{L I_{i}}\right)_{n}^{\left(K, x_{L}, a\right)}=G_{n}^{\left(K, x_{L}, a\right)} \\
=\frac{K}{\mathcal{S}+G_{(n-1)}(\mathcal{S})} \\
n \text { odd } \\
\left(\frac{I_{o}}{L V_{i}}\right)_{n}^{\left(K, x_{L}, a\right)}=G_{n}^{\left(K, x_{L}, a\right)} \\
=\frac{K}{\mathcal{S}+G_{(n-1)}(\mathcal{S})}\end{array}$ & $\begin{array}{l}G_{n}^{\left(K, x_{L}\right)}(\mathcal{S}) \\
=\frac{K \operatorname{den}_{n-1}^{\left(K, x_{L}, a\right)}(\mathcal{S})}{\mathcal{S} \operatorname{den}_{n-1}^{\left(K, x_{L}, a\right)}(\mathcal{S})+\operatorname{num}_{n-1}^{\left(K, x_{L}, a\right)}(\mathcal{S})}\end{array}$ \\
\hline
\end{tabular}

All currents through each inductor and voltages through each capacitor are determined by their corresponding FWFs. For $n=40$ of RL-FEC-Losses in Fig. 13, its general model generated with all FWFs for all currents in each inductor and all voltages in each capacitor in Fig. 14.

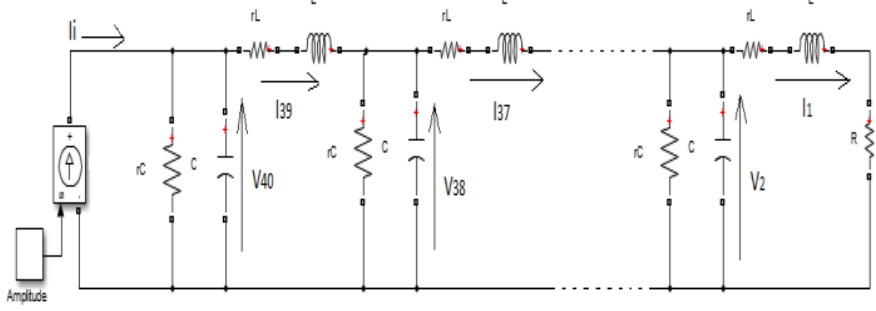

Fig. 13. $n^{\text {th }}$ even order RL-FEC-Losses.
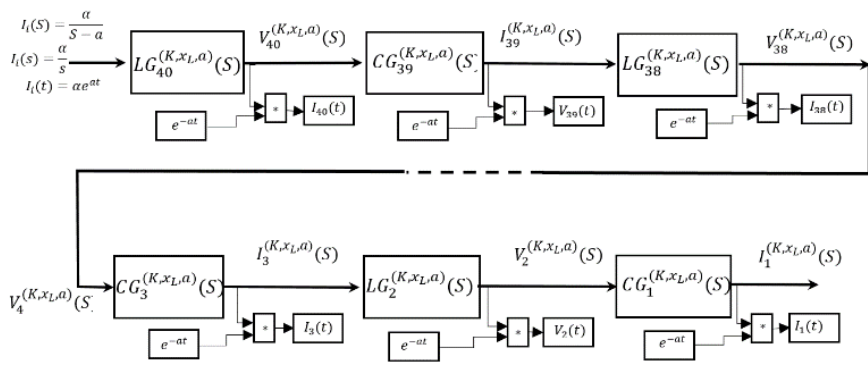

Fig. 14. $n^{\text {th }}=40$ even order RL-FEC-Losses Model using FWFs for each current and voltage branch in reference $\mathcal{L}(\mathcal{S}, a)$.

\section{Comparative Simulation OF RC-FEC-LOSSES AND THEIR FWFS}

Comparative simulation studies were conducted between the electrical circuits using MATLAB-Simulink model with Fibonacci wave functions for each order in Table I. The studies confirm that these electrical LC ladder circuits for different orders representing different transmission lines length with losses defined by $r_{L}$ and $r_{C}$ follow the same recurrent Fibonacci sequence modelled by FWFs [1].

Case 1: $R=1 \Omega ; L=1 H ; C=1 F ; r_{L}=0.02 \Omega ; r_{C}=\frac{L}{r_{L} C}=50 \Omega$

In this case $\left(K, x_{c}, a\right)=(1,1,0.2)$. Pascal's Triangle in Table II presented in [1] will be used to determine all FWFs.

$G_{14}^{\left(K, x_{c}, a\right)}(\mathcal{S})$ taken as example is an even function, using its numerator and denominator coefficients are expressed in (15) using Table *II in reference $\mathcal{L}(\mathcal{S}, a)$.

$$
\begin{aligned}
& \left(\frac{I_{o}}{C V_{i}}\right)_{14}^{\left(K, x_{c}, a\right)}=G_{14}^{\left(K, x_{c}, a\right)}(\mathcal{S})=\frac{\operatorname{Kden}_{13}^{\left(K, x_{c}, a\right)}(\mathcal{S})}{\operatorname{den}_{14}^{\left(K, x_{c}, a\right)}(\mathcal{S})} \\
& \operatorname{den}_{13}^{\left(K, x_{c}, a\right)}(\mathcal{S})=1 s^{13}+1 x_{c} \mathcal{S}^{12}+12 K \mathcal{S}^{11}+11 K x_{c} \mathcal{S}^{10} \\
& +55 K^{2} \mathcal{S}^{9}+45 K^{2} x_{c} \mathcal{S}^{8}+120 K^{3} \mathcal{S}^{7} \\
& +84 K^{3} x_{c} \mathcal{S}^{6}+126 K^{4} \mathcal{S}^{5}+70 K^{4} x_{c} \mathcal{S}^{4} \\
& +56 K^{5} \mathcal{S}^{3}+21 K^{5} x_{c} \mathcal{S}^{2}+7 K^{6} \mathcal{S} \\
& +1 K^{6} x_{c} \\
& \operatorname{den}_{14}^{\left(K, x_{c}, a\right)}(\mathcal{S})=1 s^{14}+1 x_{c} \mathcal{S}^{13}+13 K \mathcal{S}^{12}+12 K \boldsymbol{x}_{c} \mathcal{S}^{11} \\
& +66 K^{2} \mathcal{S}^{10}+55 K^{2} x_{c} \mathcal{S}^{9}+165 K^{3} \mathcal{S}^{8} \\
& +120 K^{3} x_{c} \mathcal{S}^{7}+210 K^{4} \mathcal{S}^{6} \\
& +126 K^{4} x_{c} \mathcal{S}^{5}+126 K^{5} \mathcal{S}^{4} \\
& +56 K^{5} x_{c} \mathcal{S}^{3}+28 K^{6} \mathcal{S}^{2}+7 K^{6} x_{c} \mathcal{S} \\
& +1 K^{7}
\end{aligned}
$$

TABLE III: PASCAL'S TRIANGLE GENERAL FORM WITH MULTIPLICATION

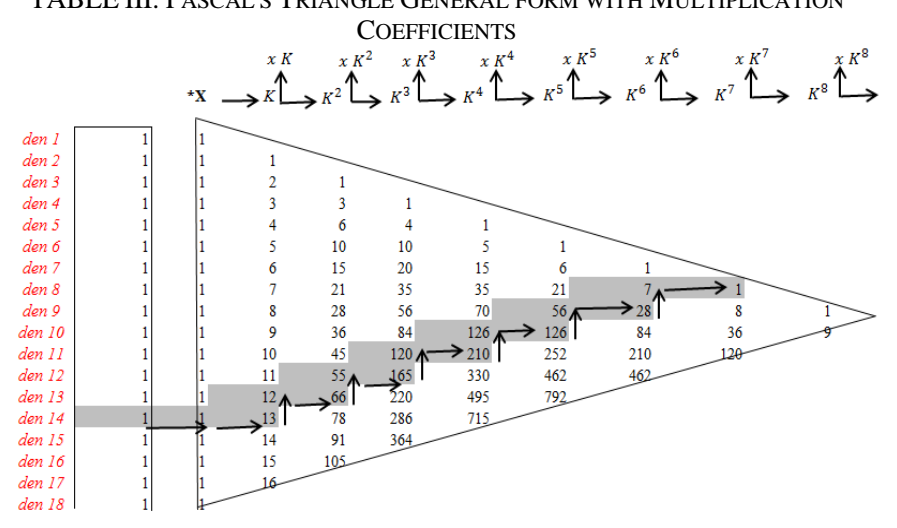

Using input voltage $V_{i}(t)=\alpha$ for all even orders FWFs and input current $I_{i}(t)=\alpha$ for all odd orders FWFs. Fig. 15 shows the block diagram in both references $\mathcal{L}(s)$ and $\mathcal{L}(\mathcal{S}, a)$ for voltage as input, the figure is also applicable when the input is current. Simulation will be used in $\mathcal{L}(\mathcal{S}, a)$ since all FWFs are perfectly known.
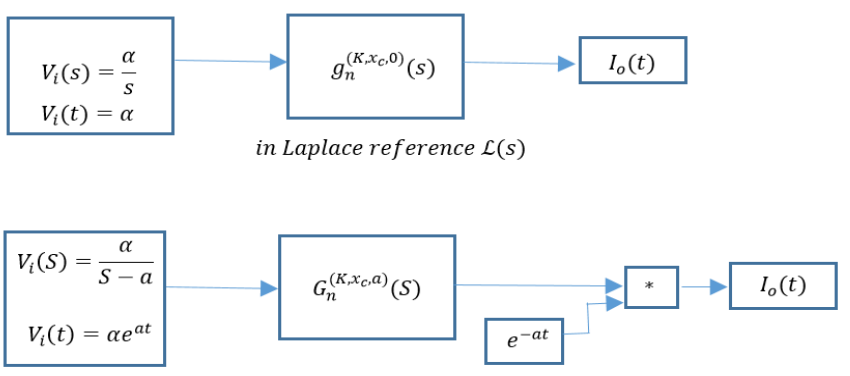

in Laplace reference $\mathcal{L}(S, a)$

Fig. 15. FWFs block diagram in Laplace $\mathcal{L}(s) \& \mathcal{L}(\mathcal{S}, a)$. 
This block diagram is also applicable for all odd orders FWFs with current as input and voltage as output.

Simulations of $\left(\frac{I_{O}}{V_{i}}\right)_{40}^{(1,1,0.2)}=C * G_{40}^{(1,1,0.2)}(\mathcal{S})$ model and RCFEC-losses Simulink model for order 40 shown in figure 16 are identical with input voltage $V_{i}(t)=\alpha=1 \mathrm{~V}$.

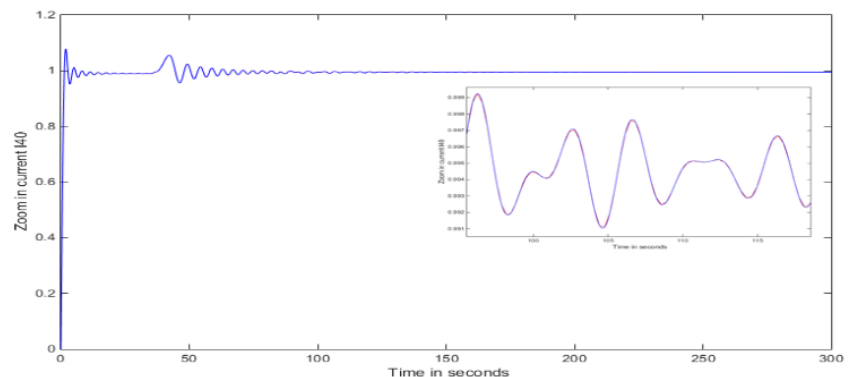

Fig. 16. RC-FEC-Losses $\left(\frac{I_{o}}{V_{i}}\right)_{40}^{(1,1,0.2)}=C * G_{40}^{(1,1,0.2)}(\mathcal{S}) \&$ Simulink circuit Model.

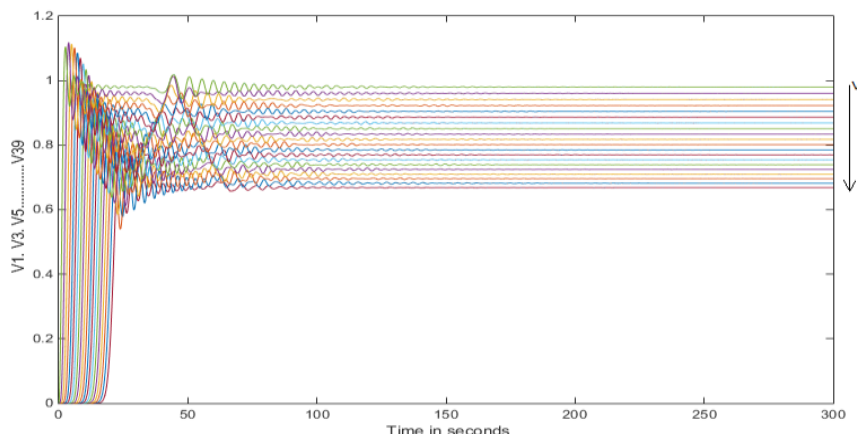

Fig. 17. $n^{\text {th }}=40$ RC-FEC-Losses for all voltages with FWFs \& Simulink circuit model.

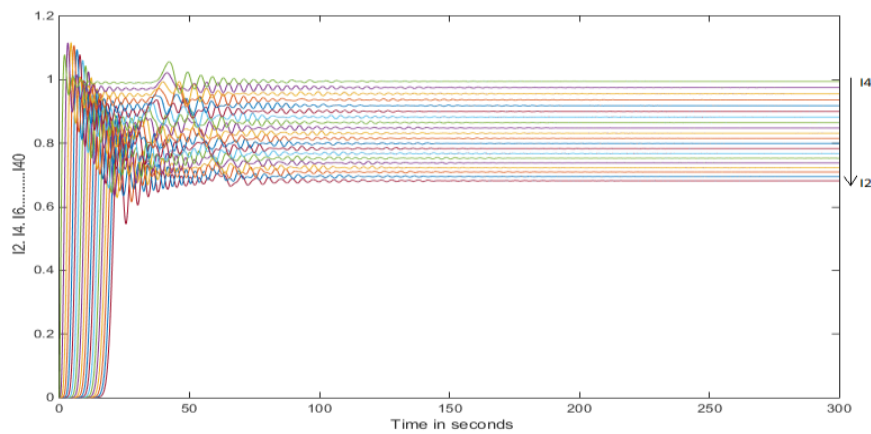

Fig. 18. $n^{\text {th }}=40$ RC-FEC-Losses for currents with FWFs \& Simulink circuit model.

Steady states values (SSV) can be easily found using Table IV below with $\left(K, x_{c}, a\right)=(1,1,0.2)$.

TABLE IV: RC-FEC-LOSSES FWFS SYSTEM GAIN $(\mathcal{S} \rightarrow a)$

\begin{tabular}{|c|c|}
\hline $\begin{array}{l}\left(\frac{V_{o}}{L I_{i}}\right)_{1}^{\left(K, x_{c}, a\right)}=G_{1}^{\left(K, x_{c}, a\right)} \\
=\frac{K}{a+x_{c}}\end{array}$ & $\left(\frac{V_{o}}{L I_{i}}\right)_{1}^{\left(K, x_{c}\right)}=G_{1}^{\left(K, x_{c}, a\right)}=\frac{K}{1 a+1 x_{c}}$ \\
\hline $\begin{array}{l}\left(\frac{I_{o}}{C V_{i}}\right)_{2}^{\left(K, x_{c}, a\right)} \\
=G_{2}^{\left(K, x_{c}, a\right)} \\
=\frac{K}{a+\frac{K}{a+x_{c}}}\end{array}$ & $\begin{aligned}\left(\frac{I_{o}}{C V_{i}}\right)_{2}^{\left(K, x_{c}, a\right)}=G_{2}^{\left(K, x_{c}, a\right)} & \\
& =\frac{K a+K \boldsymbol{x}_{\boldsymbol{c}}}{1 a^{2}+1 \boldsymbol{x}_{\boldsymbol{c}} a+1 \boldsymbol{K}}\end{aligned}$ \\
\hline $\begin{array}{l}\left(\frac{V_{o}}{L I_{i}}\right)_{3}^{\left(K, x_{c}, a\right)}=G_{3}^{\left(K, x_{c}, a\right)} \\
=\frac{K}{a+\frac{K}{a+\frac{K}{a+x_{c}}}}\end{array}$ & $\begin{array}{l}\left(\frac{V_{o}}{L I_{i}}\right)_{3}^{\left(K, x_{c}, a\right)}=G_{3}^{\left(K, x_{c}, a\right)} \\
=\frac{K a^{2}+K a \boldsymbol{x}_{\boldsymbol{c}}+K^{2}}{1 a^{3}+1 \boldsymbol{x}_{\boldsymbol{c}} a^{2}+2 \boldsymbol{K} a+1 \boldsymbol{K} \boldsymbol{x}_{\boldsymbol{c}}}\end{array}$ \\
\hline
\end{tabular}

\begin{tabular}{|c|c|}
\hline $\begin{array}{l}\left(\frac{I_{o}}{C V_{i}}\right)_{4}^{\left(K, x_{c}, a\right)} \\
=G_{4}^{\left(K, x_{c}, a\right)} \\
=\frac{K}{a+\frac{K}{a+\frac{K}{a+\frac{K}{a+x_{c}}}}}\end{array}$ & $\begin{array}{l}\left(\frac{I_{o}}{C V_{i}}\right)_{4}^{\left(K, x_{c}, a\right)}=G_{4}^{\left(K, x_{c}, a\right)} \\
=\frac{K a^{3}+K \boldsymbol{x}_{\boldsymbol{c}} a^{2}+2 K^{2} a+K^{2} \boldsymbol{x}_{\boldsymbol{c}}}{1 a^{4}+1 \boldsymbol{x}_{\boldsymbol{c}} a^{3}+3 \boldsymbol{K} a^{2}+2 \boldsymbol{K} \boldsymbol{x}_{\boldsymbol{c}} a+1 \boldsymbol{K}^{2}}\end{array}$ \\
\hline $\begin{array}{l}\left(\frac{I_{o}}{C V_{i}}\right)_{n}^{\left(K, x_{c}, a\right)}=G_{n}^{\left(K, x_{c} \cdot a\right)} \\
=\frac{K}{a+G_{n-1}^{\left(K, x_{c}, a\right)}(a)} \\
n \text { even } \\
\left(\frac{V_{o}}{L I_{i}}\right)_{n}^{\left(K, x_{c}\right)}=G_{n}^{\left(K, x_{c}, a\right)} \\
=\frac{K}{a+G_{n-1}^{\left(K, x_{c}, a\right)}(a)} \\
n \text { odd }\end{array}$ & $\begin{array}{l}G_{n}^{\left(K, x_{c}, a\right)}(a) \\
=\frac{K \operatorname{den}_{n-1}^{\left(K, x_{c}, a\right)}(a)}{\operatorname{aden}_{n-1}^{\left(K, x_{c}, a\right)}(a)+\operatorname{num}_{n-1}^{\left(K, x_{c}, a\right)}(a)} \\
G_{n}^{\left(K, x_{c}, a\right)}(a)=\frac{K \operatorname{den}_{n-1}^{\left(K, x_{c}, a\right)}(a)}{\operatorname{den}_{n}^{\left(K, x_{c}, a\right)}(a)}\end{array}$ \\
\hline
\end{tabular}

\section{TRANSMISSION LINES WITH LOSSES IN SHORT AND OPEN LOAD}

In radio frequency telecommunication field, analyzing transmission cables quality is crucial in RF design. Voltage Standing Wave Ratio (VSWR) return losses (RL) and distance to fault (DTF) are the main three ways to check the transmission cable quality and to detect any damage issues. The analysis performed with antenna termination, load termination, short and open load termination. In this paragraph, Fibonacci wave functions FWFs will be determined in both open and short transmission cable termination and compared with recurrent RC-FEC-losses Simulink model order 40 in Fig. 12.

The input impedance or admittance can be found using Pascal's triangle for the case $R=1 \Omega ; L=1 H ; C=1 F ; r_{L}=$ $0.02 \Omega ; r_{C}=\frac{L}{r_{L} C}=50 \Omega$.

This is a particular case of Pascal's triangle general form detailed in [1].

Below is an example of $G_{13}(S)$ and $G_{14}(S)$ using Pascal's triangle in Table III.

For $13^{\text {th }}$ and $13^{\text {th }}$ order RL-FEC-Losses circuit in Fig. 19 is used to determine the corresponding FWFs.

$$
\begin{gathered}
\left(\frac{I_{o}}{C V_{i}}\right)_{13}^{\left(K, x_{L}, a\right)}=G_{13}^{\left(K, x_{L} \cdot a\right)}(\mathcal{S})=\frac{\operatorname{Kden}_{12}^{\left(K, x_{L}, a\right)}(\mathcal{S})}{\operatorname{den}_{13}^{\left(K, x_{L}, a\right)}(\mathcal{S})} \\
\left(\frac{V_{o}}{L I_{i}}\right)_{14}^{\left(K, x_{L}, a\right)}=G_{14}^{\left(K, x_{L}, a\right)}(\mathcal{S})=\frac{\operatorname{Kden}_{13}^{\left(K, x_{L}, a\right)}(\mathcal{S})}{\operatorname{den}_{14}^{\left(K, x_{L}, a\right)}(\mathcal{S})} \\
x_{L}=\frac{R}{L}
\end{gathered}
$$

For RC-FEC-Losses circuit in Fig. 16 for order 13 and 14.

$$
\begin{gathered}
\left(\frac{I_{o}}{C V_{i}}\right)_{14}^{\left(K, x_{c}, a\right)}=G_{14}^{\left(K, x_{c}, a\right)}(\mathcal{S})=\frac{\operatorname{Kden}_{13}^{\left(K, x_{L}, a\right)}(\mathcal{S})}{\operatorname{den}_{14}^{\left(K, x_{c}, a\right)}(\mathcal{S})} \\
\left(\frac{V_{o}}{L I_{i}}\right)_{13}^{\left(K, x_{c}, a\right)}=G_{13}^{\left(K, x_{c}, a\right)}(\mathcal{S})=\frac{K \operatorname{den}_{12}^{\left(K, x_{c}, a\right)}(\mathcal{S})}{\operatorname{den}_{13}^{\left(K, x_{c}, a\right)}(\mathcal{S})} \\
x_{c}=\frac{1}{R C}
\end{gathered}
$$


For RC-FEC-Losses or RL-FEC-Losses, the input impedance or admittance for any order $n$ and for both shortcircuit and open-circuit is as follow.

For short-circuit $(R=0 \Omega), x_{c}=\infty$ using RC-FEC and $x_{L}=0$ using RL-FEC-losses. Equations (19), (20) and (21) become.

$$
\begin{aligned}
& \left(\frac{V_{o}}{L I_{i}}\right)_{14}^{\left(k, x_{L}=0, a\right)}=G_{14}^{\left(k, x_{L}=0, a\right)}(\mathcal{S}) \\
& 1 \mathcal{S}^{13}+12 K \mathcal{S}^{11}+55 K^{2} \mathcal{S}^{9}+120 K^{3} \mathcal{S}^{7}
\end{aligned}
$$

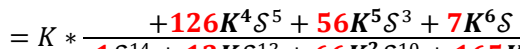

$$
\begin{aligned}
& \begin{array}{l}
1 \delta^{14}+13 K \mathcal{S}^{12}+66 K^{2} \mathcal{S}^{10}+165 K^{3} \mathcal{S}^{8} \\
+210 K^{4} S^{6}+126 K^{5} S^{4}+28 K^{6} S^{2}+1 K^{7}
\end{array} \\
& \left(\frac{I_{o}}{C V_{i}}\right)_{13}^{\left(k, x_{L}=0, a\right)}=G_{13}^{\left(k, x_{L}=0, a\right)}(\mathcal{S}) \\
& =K * \frac{1 \mathcal{S}^{12}+11 K \mathcal{S}^{10}+45 K^{2} \mathcal{S}^{8}+84 K^{3} \mathcal{S}^{6}}{+70 K^{4} \mathcal{S}^{4}+21 K^{5} \mathcal{S}^{2}+1 K^{6}} \\
& \left(\frac{I_{o}}{C V_{i}}\right)_{14 c}^{\left(K, x_{C}=\infty, a\right)}=G_{14}^{\left(K, x_{C}=\infty, a\right)}(s) \\
& =K * \frac{1 \mathcal{S}^{12}+11 K \mathcal{S}^{10}+45 K^{2} \mathcal{S}^{8}+84 K^{3} \mathcal{S}^{6}}{+70 K^{4} \mathcal{S}^{4}+21 K^{5} \mathcal{S}^{2}+1 K^{6}} \\
& \left(\frac{V_{o}}{L I_{i}}\right)_{13 c}^{\left(K, x_{C}=\infty, a\right)}=G_{13}^{\left(K, x_{C}=\infty, a\right)}(\mathcal{S}) \\
& =K * \frac{1 \mathcal{S}^{11}+10 K \mathcal{S}^{9}+36 K^{2} \mathcal{S}^{7}}{1 \mathcal{S}^{3} \mathcal{S}^{5}+35 K^{4} \mathcal{S}^{3}+6 K^{5} \mathcal{S}^{1}}
\end{aligned}
$$

For an open-circuit $(R=\infty . \Omega), x_{c}=0$ using RC-FECLosses and for RL-FEC-Losses $x_{L}=\infty$. Equations are determined below.

$$
\begin{aligned}
& \left(\frac{I_{o}}{C V_{i}}\right)_{14}^{\left(K, x_{C}=0, a\right)}=G_{14}^{\left(K, x_{C}=0, a\right)}(\mathcal{S}) \\
& =K * \frac{1 \mathcal{S}^{13}+12 K \mathcal{S}^{11}+55 K^{2} \mathcal{S}^{9}+120 K^{3} \mathcal{S}^{7}}{+126 K^{4} \mathcal{S}^{5}+56 \boldsymbol{K}^{5} \mathcal{S}^{3}+7 \boldsymbol{K}^{6} \mathcal{S}} \\
& =K * \frac{+126 K^{4} \mathcal{S}^{5}+56 K^{5} \mathcal{S}^{3}+7 K^{6} \mathcal{S}}{1 \mathcal{S}^{14}+13 K \mathcal{S}^{12}+66 K^{2} \mathcal{S}^{10}+165 K^{3} \mathcal{S}^{8}} \\
& +210 K^{4} \mathcal{S}^{6}+126 K^{5} \mathcal{S}^{4}+28 K^{6} \mathcal{S}^{2}+1 K^{7} \\
& \left(\frac{V_{o}}{L I_{i}}\right)_{13}^{\left(K, x_{C}=0, a\right)}=G_{13}^{\left(K, x_{C}=0, a\right)}(\mathcal{S}) \\
& 1 \mathcal{S}^{12}+11 K \mathcal{S}^{10}+45 K^{2} \mathcal{S}^{8}+84 K^{3} \mathcal{S}^{6} \\
& =K * \frac{+70 K^{4} \mathcal{S}^{4}+21 K^{5} \mathcal{S}^{2}+1 K^{6}}{1 \mathcal{S}^{13}+12 K \mathcal{S}^{11}+55 K^{2} \mathcal{S}^{9}+120 K^{3} \mathcal{S}^{7}} \\
& +126 K^{4} \mathcal{S}^{5}+56 K^{5} \mathcal{S}^{3}+7 K^{6} \mathcal{S} \\
& \left(\frac{V_{o}}{L I_{i}}\right)_{14}^{\left(K, x_{L}=\infty, a\right)}=G_{14}^{\left(K, x_{L}=\infty, a\right)}(\mathcal{S}) \\
& =K * \frac{1 \mathcal{S}^{12}+11 K \mathcal{S}^{10}+45 K^{2} \mathcal{S}^{8}+84 K^{3} \mathcal{S}^{6}}{+70 \boldsymbol{K}^{4} \mathcal{S}^{4}+21 \boldsymbol{K}^{5} \mathcal{S}^{2}+1 \boldsymbol{K}^{6}} \\
& \left(\frac{I_{o}}{C V_{i}}\right)_{13}^{\left(K, x_{L}=\infty, a\right)}=G_{13}^{\left(K, x_{L}=\infty, a\right)}(\mathcal{S})
\end{aligned}
$$

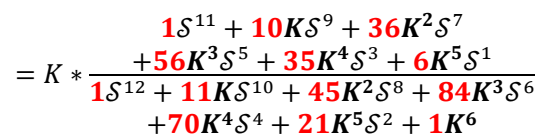

Simulations were conducted using general model shown in Fig. 7 and RC-FEC-Losses Simulink model for $n=40$ $I_{40}^{\left(1, x_{c}=0,0.2\right)}, V_{21}^{\left(1, x_{c}=0,0.2\right)}$ are perfectly the same (Fig. 19 and 20).

All current across inductors and all voltages across capacitors are also shown for both FWFs and Simulink model for RC-FEC-Losses order 40 in Fig. 21 and 22.

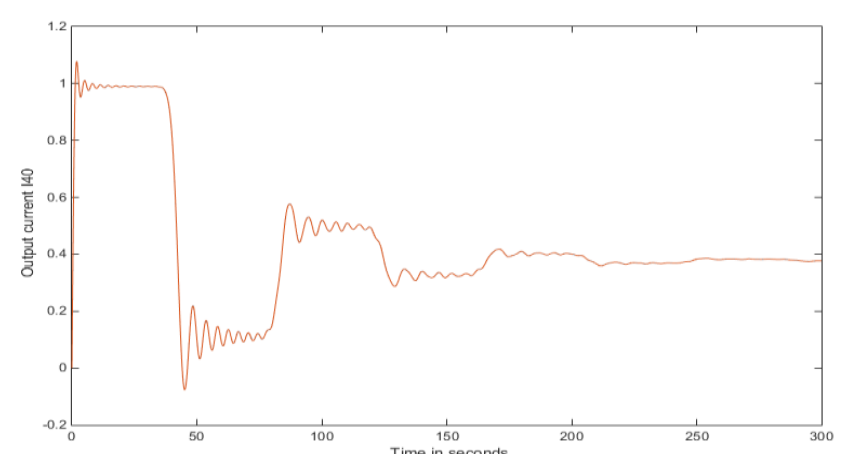

Fig. 19. RC-FEC-Losses and general model $\left(I_{40}^{(1,0,0.2)}, V_{i}=1 V, R=\infty \Omega\right)$.

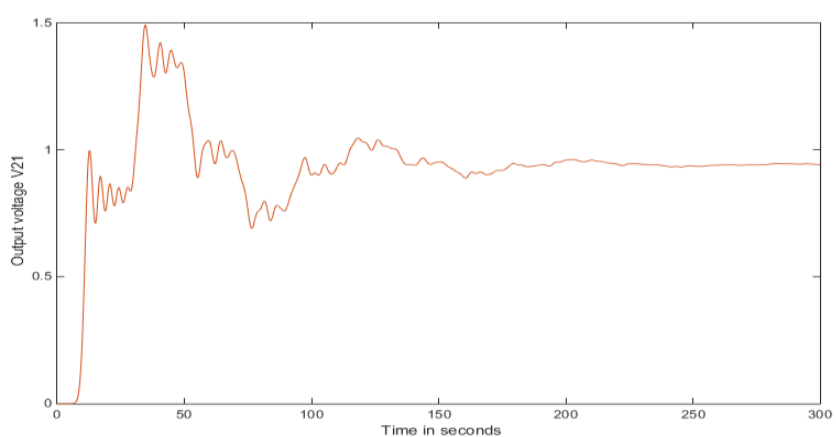

Fig. 20. RC-FEC-Losses and general model $\left(V_{21}^{(1,0,0.2)}, V_{i}=1 V, R=\infty \Omega\right)$.

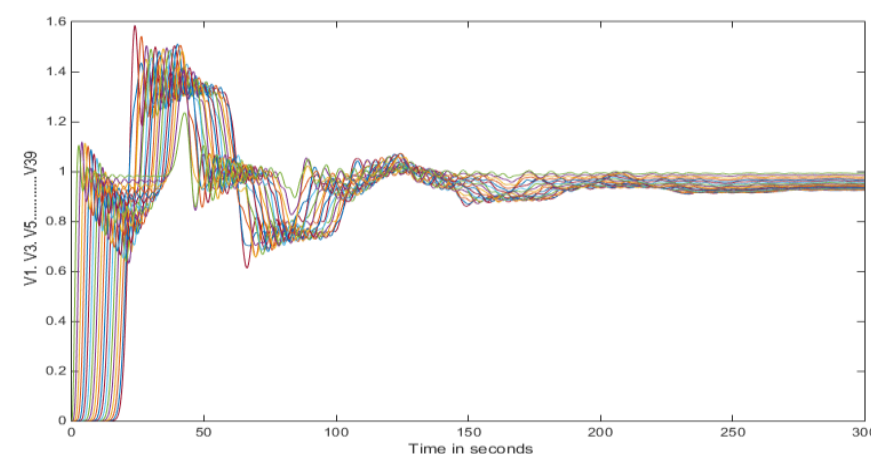

Fig. 21. RC-FEC-Losses and general model $\left(V_{1,3,5, \ldots, 39}^{(1,0,0.2)}, V_{i}=1 V, R=\infty \Omega\right)$.

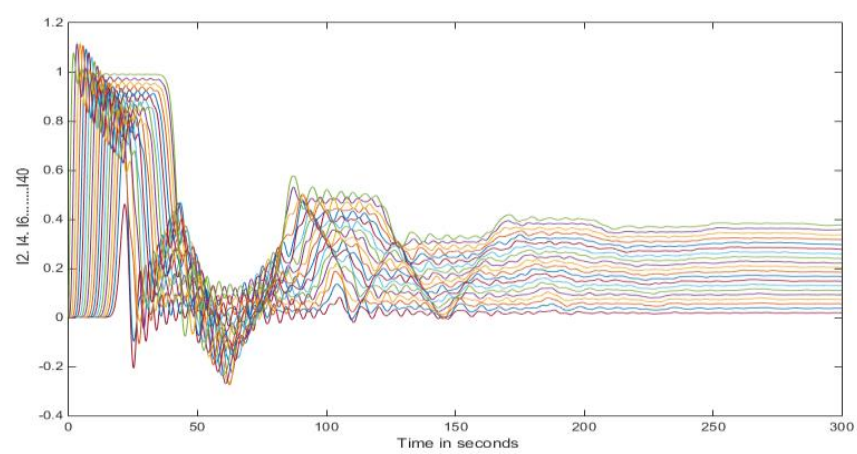

Fig. 22. RC-FEC-Losses and general model $\left(I_{2,4,6, \ldots, 40}^{(1,0,0.2)}, V_{i}=1 V, R=\infty \Omega\right)$.

\section{CONCLUSION}

In this paper, a complete Fibonacci wave functions FWFs model that was first applied to an LC ladder network representing lossless transmission line [1] and [2] is extended to LC ladder network presenting losses $\left(r_{c}, r_{L}\right)$ to model a transmission line with no distortion subject to Heaviside condition. The general model with losses proposed in this paper helps find automatically all transfer functions, any input impedance or admittance for any recurrent LC network order. 
This research shows the importance of Fibonacci wave functions and their application in modelling recurrent complicated systems. These FWFs model research is still ongoing to model LC ladder network presenting losses $\left(r_{c}, r_{L}\right)$ with distortion non subject to Heaviside condition.

\section{REFERENCES}

[1] S. Hissem, Mamadou Lamine Doumbia, "New Fibonacci Recurrent Systems Applied to Transmission lines Input Impedance and Admittance", IEEE International Conference Advances in Science and Engineering Technology (ASET), March 2019.

[2] S. Hissem, Mamadou Lamine Doumbia, "Infinite Spring-Mass Chain Model Using Fibonacci Wave Functions", IEEE International Conference Advances in Science and Engineering Technology (ASET), March 2019.

[3] Josha W. Phinney, David J. Perreault and Jeffrey H. Lang, "Synthesis of Lumped Tansmission Line Analogs", $37^{\text {th }}$ IEEE Power Electronics Specialists Conference, June 2006, pp. 2967-2978.

[4] Antonije R. Djordjevic, Alenka G. Zajic, Dejan V. Tosic, and Truc Hoang, "A Note on the Modelling Transmission-line Losses", Transactions on microwave theory and technics, Vol. 51, No. 2, pp. 483-486, February 2003.

[5] Ana Flavia G. Greco, Joaquim J. Barroso, Jose O. Rossi, "Modelling and Analysis of Ladder-Network Transmission Lines with Capacitive and Inductive Lumped Elements", Journal of Electromagnetic Analysi and Applications, 2013, 5, pp. 213-218.

[6] Ana Flávia G. Greco, Joaquim J. Barroso and José O. Rossi, "Modeling and Analysis of Ladder-Network Transmission Lines with Capacitive and Inductive Lumped Elements", Journal of Electromagnetic Analysis and Applications, 2013, 5, 213-218.

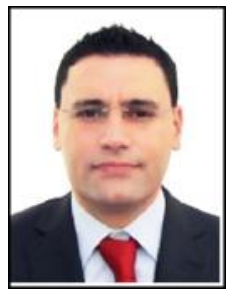

Simon Hissem received the B.S. degree in electronics in 1989 and the M.S. degree in industrial electronics in 1995 and $\mathrm{PhD}$ degree in electrical engineering from Trois-Rivières University, Trois- Rivières, Québec, Canada in 2019.

Dr. Simon has more than 15 years industrial experience in telecommunication field. He was working as Senior RF telecommunication design engineer in different countries: in North America with Telus Mobility in Montreal, Québec, Canada; with Sprint-Nextel in Boston, Massachusetts, USA; in Europe with Forsk Telecom in Toulouse, France; in North Africa with Wataniya Telecom in Algiers, Algeria. Since 2013, Dr. Simon is working in United Arab Emirates with Higher College of Technology as Electrical and Electronic Engineering faculty. His research interests include system modelling, control systems and signal processing.

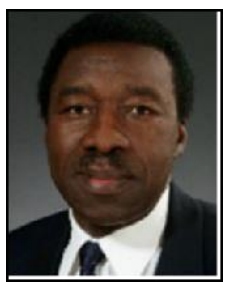

Mamadou Lamine Doumbia received the M.Sc. degree in electrical engineering from Moscow Power Engineering Institute (Technical University), Moscow, Russia, in 1989, the M.Sc. degree in industrial electronics from the Université du Québec à Trois-Rivières (UQTR), Trois-Rivieres, QC, Canada in 1994, and the $\mathrm{Ph} . \mathrm{D}$. degree in electrical engineering from the Ecole Polytechnique de Montreal, Montreal, QC, Canada, in 2000. From 2000 to 2002, he was a Lecturer at the Ecole Polytechnique de Montreal and the CEGEP SaintLaurent, Montreal, QC, Canada. He was a Senior Research Engineer (20022003) with the CANMET Energy Technology Centre (Natural Resources Canada) and a Postdoctoral Researcher (2003-2005) with the Hydrogen Research Institute (HRI). Since 2005, he has been a Professor with the Department of Electrical and Computer Engineering, UQTR. He has authored or coauthored more than 80 papers in international journals and conferences. His research interests include renewable energy systems, distributed energy resources, electric drives, power electronics, and power quality. Dr. Doumbia is a member of the HRI, a member of the IEEEPower Electronics Society. He is a Professional Engineer and a member of the Ordre des Ingenieurs du Québec. He is currently an editorial board member of International Journal of Renewable Energy Research and International Journal of Smart Grid. 